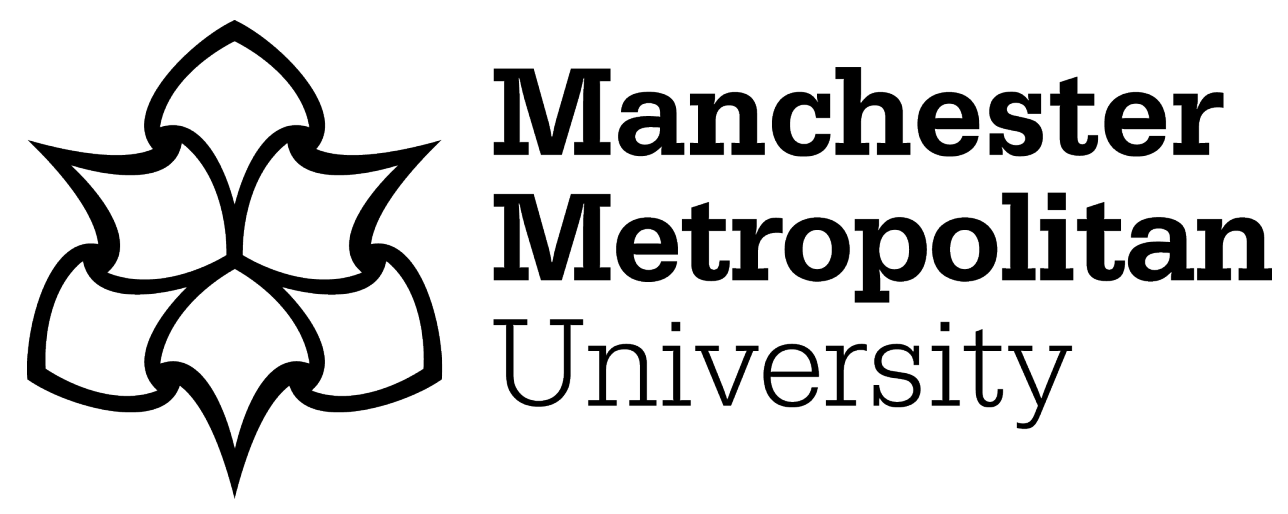

Jones, P and Skinner, H (2014) E-learning globalization: the impact of elearning - what difference has it made? Education and Training, $56(2 / 3)$. ISSN 0040-0912

Downloaded from: https://e-space.mmu.ac.uk/621231/

Version: Accepted Version

Publisher: Emerald

DOI: https://doi.org/10.1108/ET-11-2013-0128

Please cite the published version 


\section{E-learning globalization: the impact of e- learning - what difference has it made?}

\section{Citation:}

Jones Paul, Skinner Heather, (2014) "E-learning globalization: the impact of e-learning - what difference has it made?", Education + Training, Vol. 56 Issue: 2/3, https://doi-org.ezproxy.mmu.ac.uk/10.1108/ET-11-2013-0128

The ubiquity of information and communication technology in the past two decades or so, and the more recent growth in the use of mobile wireless devices, has transformed various aspects of daily life for many people worldwide. It is therefore unsurprising that such technologies would also find their way into transforming various aspects of education and training. What may be surprising is that, even though forms of computer assisted learning have been used for over 40 years, educators and trainers can still find themselves grappling with relatively basic issues of defining the way this transformation occurs, or indeed if and how it should occur. Although there are many other terms in use, such as blended learning, hybrid learning, technology enhanced learning and so on, the term e-learning is the one used for the title and focus of this special issue as it can be seen to encompass all aspects of learning using all aspects of electronic technologies. While some definitional issues may appear in some of the papers in this special issue, the overall focus of this special issue is to move the debate forward from such more basic matters. In particular, while we recognise that there is a significant body of literature which has espoused the effective construction of e-learning pedagogy and programmes in the previous decade, the focus of this special edition is to provide evidence to support the proposition that e-learning has been a beneficial and transformational innovation. Therefore, this special issue considers issues of impact rather than issues of definition. At its inception, one of the proposed benefits of elearning was seen to be its ability to educate and train learners at a distance. Thus this special issue has also sought to consider issues of impact in terms of the globalisation of e-learning.

The first paper in the special edition by Singh and Hardaker considers barriers and enablers to the adoption and diffusion of e-learning. The study identifies that the importance of individual factors influencing the adoption of e-learning has been acknowledged by prior studies, and the underlying message has emerged that levels of e-learning adoption would be higher if strategic managers recognised the social dimensions of e-learning innovation. The argument is that currently e-learning is geared towards technically 'literate' and innovative staff, and this strategy reduces the likelihood of mainstream faculty actually adopting instructional technology for their own teaching.

The second paper, authored by Sloan, Porter, Robins and McCourt, focuses on the challenges presented in learning and teaching on how to support international postgraduate students undertaking their dissertations with the assistance of an elearning tool. The tool evolved into an e-learning resource supporting student understanding of the content, structure, planning and writing of a dissertation. The e-learning tool focused on assisting international students understand what the 
generic contents of each chapter of a dissertation should contain and supporting them in engaging in research as a transferable skill.

Goulding and Khuzzan's study examines the use, construct and pervasiveness of learning styles theory in an e-learning context. Evidence identified that the more thoroughly instructors understand the differences in learning styles, the better chance they had of meeting the diverse learning needs of their learners. The Diagnostic Questionnaire utilised in this study was used to explore different approaches to use in different learning environments. Specifically, it allows training providers to understand the nuances and dependencies associated with learner styles, behaviour, learner effectiveness and motivation. This study provides a new understanding on the learning process and how this links to e-learning pedagogy and learning styles. It presents a mechanism for embedding a Diagnostic Questionnaire into a Personalised e-Learning Environment.

McGregor, Shaw, Sinclair, Sutherland, Munro and Ross consider the development and implementation of a national online learning initiative for a cancer treatment system highlighting the rigorous approach undertaken for the delivery of a quality evidence-based resource for the professional development of all health professionals involved in the delivery of cancer care. The study recognised that time constraints of health professionals continue to be a major barrier to sustained online learning participation. The paper by Leeds explores the experience of elearners in relation to their time preferences to examine how technology has changed temporal culture in e-learning. The study found e-learners may experience temporal culture shock when starting to study online for the first time. It highlights the need to explore an individual's time preference and to consider the temporal culture of the e-learning programme. Dickfos, Cameron and Hodgson describe the evolution of a blended learning strategy in a company law course for accounting students, and evaluate its impact on assessment and student self-reflection. The study identifies the benefits of and likely issues facing educators when considering the deployment of blended learning technologies to teach and assess oral communication skills. The study contributes to pedagogy by describing the innovative use of video cameras in assessing elevator pitches and extends the literature on video presentations in higher education, in particular, its positive influence on student self-reflection.

Lean, Moizer and Newbery's paper describes an approach for utilising a critical incident method within the context of an online business simulation game in order to provide an effective framework for reflective learning. The paper provides educators with a blended learning method that can be employed to enhance the impact of e-learning through structured reflection. Smith and Barrett's study considers the use of an informal online discussion forum (ODF) to encourage voluntary participation and promote double-loop learning by small business owners (SBOs). This research provided evidence that an ODF for SBOs supported double-loop deep learning; although participation could not be assumed simply by the online availability of the discussion resource. The ninth and final paper in this special issue was authored by Wong, Tatnall and Burgess and explores a framework of blended learning to assess the readiness, intensity of adoption and impact of blended learning systems. 
In conclusion, it is apparent from the papers received that e-learning systems are making a considerable impact on education and training for adults. A review of the papers included within the special issues reveals e-learning systems having a range of impacts and deployments. This included the assessment of the effectiveness of full e-learning systems and also hybrid blended learning programmes. Blended learning systems are now becoming integrated across the educational sectors with commonplace use of virtual learning environments to support and enhance the student experience. Moreover, such systems are regularly used by the teaching community to support and enhance their pedagogical practices and assessment strategies in areas such as business simulation and plagiarism identification. Within this special issue, a range of different users and processes were impacted upon and supported effectively including consideration of learning styles, time management issues, self-reflection, dissertation completion impacting upon the traditional and part time student community, medical professionals in cancer care and the small business community. The future for e-learning, particularly within the university sector, is an exciting one with increased sophistication of provision and greater deployment of mobile learning technologies to provide customised individual learning solutions.

This special issue has proved a demanding but rewarding process and we would like to take this opportunity to thank a number of key individuals who have enabled its development and delivery. First, we would like to extend our gratitude to Dr Martin McCracken, the Journal Editor of Education+Training, for his advice and patience during the development of the special edition. Moreover, we would like to extend our sincere thanks to the many individuals involved in, and who contributed to, the delivery of this issue of the special issue including the authors and referees for their time and hard work. Finally, our sincere thanks to the employees of the Emerald Publishing Group, particularly Sophie Barr, for her administrative support and efficiency throughout this process. Their commitment and dedication to the special issue of the journal is greatly appreciated. 